\title{
Análise da eficiência e o posicionamento do ranking das cooperativas de crédito do Brasil
}

\author{
Efficiency analysis and positioning of ranking of credit unions in Brazil \\ Eficiencia de análisis y posicionamiento de clasificación de credito cooperativa \\ de Brasil
}

Analyse d'efficacité et de positionnement de classement de credit cooperatif du Brésil

\author{
Fábio José Diel* \\ (fdiel@al.furb.br) \\ Tarcísio Pedro da Silva* \\ (tarcisio@furb.br)
}

Recebido em 05/02/2013; revisado e aprovado em 25/04/2013; aceito em 12/06/2013

\begin{abstract}
Resumo: O objetivo desta pesquisa foi analisar a eficiência e o posicionamento do ranking das cooperativas de crédito do Brasil, tendo característica descritiva e documental, com tratamento quantitativo. Nos resultados, quando utilizada matriz de preferência no método AHP, o ranking original apresentado pelo BACEN altera posição de 48 das 50 cooperativas, classificando inversamente ao ranking original.

Palavras-chave: Eficiência. Ranking. Cooperativas de crédito.
\end{abstract}

Abstract: This research aimed at analyzing ranking efficiency and positioning of credit unions in Brazil, by means of documental and descriptive quantitative treatment. The results show that, when AHP matrix is preferably used, original ranking prescribed by BACEN changes the position of 48 unions out of 50 , inversely classifying to original ranking.

Key words: Efficiency. Ranking. Credit unions.

Resumen: El objetivo de la investigación fue analizar la eficacia de la clasificación y el posicionamiento de las cooperativas de crédito en Brasil, teniendo como. Descriptiva y documental, con un tratamiento cuantitativo En los resultados, la clasificación presentada por el Banco Central, cuando se utiliza con preferencia matriz AHP tiene cambios de posición de 48 cooperativas, que apunta hacia el lado contrario de la clasificación original.

Palabras clave: Eficiencia. Clasificación. Uniones de crédito.

Résumé: L'objectif de la recherche était d'analyser l'efficacité du classement et positionnement des caisses populaires au Brésil, ayant comme. Descriptive et documentaire, d'un traitement quantitatif Dans les résultats, le classement présenté par la Banque centrale, lorsqu'il est utilisé de préférence matrice de PLA a des changements de position de 48 coopératives, pointant vers le côté inverse du classement initial.

Mots-clés: Efficacité. Classement. Coopératives de crédit.

\section{Introdução}

Cooperativas de crédito são instituições financeiras inseridas no mercado sob a forma de sociedades cooperativas com o objetivo de prestação de serviços financeiros ao associado (PINHEIRO, 2005).

Lazzarini, Bialoskorski Neto e Chaddad (1999) destacam que as cooperativas de crédito têm passado por diversas mudanças nos últimos tempos, como as mudanças na gestão financeira em decorrência de suas características organizacionais. Além das mudanças, a adequação às práticas do mercado exige alterações nas entidades, de forma que, estas consigam responder com agilidade às necessidades dos clientes, sejam cooperados ou correntistas.
Conforme Soares e Melo Sobrinho (2007), "o setor cooperativista é de singular importância para a sociedade, na medida em que promove a aplicação de recursos privados e assume os correspondentes riscos em favor da própria comunidade". A forma de atuação das cooperativas, em função do comprometimento assumido pelos cooperados, pela causa cooperativa, contribui para que as perdas financeiras sejam menores em função da baixa inadimplência no recebimento do crédito concedido.

Segundo Silva, Fiorentin e Toledo Filho (2011) consideram, as cooperativas estão inseridas em um ambiente competitivo, e a busca pela eficiência deve ser o objetivo constante da organização. Dentre esses fatores devem ser levados em conta diversos indicadores como gestão de risco e gestão de crédito.

\footnotetext{
* Universidade Regional de Blumenau (FURB), Blumenau, SC, Brasil.
} 
Diante disso, se estabeleceu como questão de pesquisa indagar: qual a eficiência e o posicionamento do ranking das cooperativas de crédito do Brasil? Com isso, esta pesquisa destaca como objetivo analisar a eficiência e o posicionamento do ranking das cooperativas de crédito do Brasil.

Como justificativa para a pesquisa, quanto à utilização dos dados das cooperativas, com relação à temática foram utilizados alguns autores como: Pinheiro (2008); Dayson et al. (2010) e Silva (2012). Com relação ao resultado apresentado, Horrigan (1968) destacou a utilidade dos indicadores financeiros, bem como dos dados contábeis no desempenho das entidades.

\section{Gestão de risco}

A gestão de risco vem sendo pesquisada com mais frequência pela necessidade das empresas em solucionar problemas anteriormente despercebidos. A gestão de risco, conforme apresentado por Fama (1972), destaca, em termos gerais, que podemos sugerir que o retorno de uma carteira pode ser analisado com base no retorno de seleção segurança (seletividade) e do retorno de risco.

No gerenciamento de risco, Baccarini e Archer (2001) descrevem a utilização de uma metodologia para a classificação de risco dos projetos realizados pelo departamento de contrato e serviços de gerenciamento em uma agência de governo na Austrália Ocidental.

Zhou e Lai (2009) destacam que a avaliação de risco de crédito tornou-se cada vez mais importante área de instituições financeiras, a recente crise financeira e implementação de Basileia II. O quantitativo foi desenvolvido modelos de scoring de crédito para ajudar gerentes de crédito avaliar risco de crédito dos clientes para várias décadas. Houve uma pequena melhora na pontuação no rigor do crédito para reduzir perda significativa, que visa melhorar as decisões dos gestores de risco, visa melhorar a decisão precisão. Os resultados mostram que todos os modelos têm precisão de classificação boa quando comparado com os métodos tradicionais.

\subsection{Operações e consumo de crédito}

Conforme Brito e Assaf Neto (2008), para as instituições financeiras, crédito significa atividade de disponibilizar um valor a um tomador de recursos sob a forma de empréstimo ou financiamento. Complementam ainda que, normalmente, a concessão de crédito envolve a expectativa de recebimento de valores disponibilizado com adicional de uma remuneração sob influência, por um determinado período de tempo.

Como parte do ambiente das instituições financeiras, na atividade de concessão de crédito, Gonçalves (2005) destaca que as cooperativas de crédito diferenciam-se das demais instituições financeiras pelo fato de cobrarem pouco a mais que o custo, já que são entidades não lucrativas, assim podendo oferecer taxas mais atrativas.

Ao destacar uma nova abordagem para o consumo de crédito no pensamento econômico e financeiro, Dauten (1954) avaliou que havia aproximação em algumas áreas da economia e finanças a partir de pontos de vista divergentes, como crédito ao consumidor, causado pelo fato de alguns estudiosos que temiam que o crescimento do consumo de crédito pudesse causar a ruína do sistema econômico. No entanto outros destacam que o consumo de crédito é um dos mais importantes fatores responsáveis para o padrão de vida elevado.

No que concerne à avaliação das taxas de juros que implicam o custo do empréstimo às empresas, Archer (1964) destacou que o gestor, na sua função, deveria fazer escolhas entre: planos, políticas e estratégias. Entre outras condições, as decisões deveriam ser feitas sob diferentes graus de informações, habitualmente classificados como as condições de certeza, risco e incerteza.

Para Robbins e Foster Jr. (1957), cabe ao gestor financeiro, no processo de gestão, o planejamento do lucro, dos recursos necessários para planejamento das vendas, análise das despesas variáveis, da base fixa, dos incrementos necessários. Além disso, devese avaliar a elasticidade do custo, revisão das projeções, consideração dos estudos de engenharia para análise estatística, de forma que pudesse apresentar um realinhamento da estrutura de gestão com análise dos custos. 
Para que conseguisse aumentar seu resultado financeiro, a empresa precisaria conciliar suas finanças com a produção. Turnovsky (1970) enfatizou que qualquer empresa, no decorrer de suas operações, deve estabelecer pelo menos três tipos de decisões distintas. Primeiro, decidir sobre um plano de produção com escolhas que possam ser perfeitamente alcançadas. Segundo, decidir quanto dinheiro precisa investir em ativos fixos e capital de giro necessário para sustentar a produção. Terceiro, decidir como este investimento deve ser de financiado.

Os três tipos de decisões contribuem, como forma de mitigar os riscos, para que o processo de operações com risco seja facilitado às partes, contribuindo sobremaneira para o gerenciamento no consumo de crédito, com finalidade de utilização alinhada com a atividade final das entidades.

\subsection{Cooperativas de crédito}

As cooperativas vêm em ascendente crescimento, e uma das ramificações são as cooperativas de crédito, apresentadas conforme o tópico abaixo de cooperativas de crédito. No Brasil, a constituição da primeira cooperativa de crédito iniciou no Estado do Rio Grande do Sul, no ano de 1902, na cidade de Nova Petrópolis, hoje denominada Pioneira/RS. Porém a estruturação da legislação aconteceu somente no ano de 1964, com a regulamentação das cooperativas de crédito, sendo enquadradas na mesma atividade econômica que os bancos comerciais, conforme estabelece a Lei n. 4.595 (BRASIL, 1964).

Conforme a resolução 2771 de 30/08/2000, normatiza-se o funcionamento de dois tipos de organização, classificando-as em cooperativas de crédito mútuo e cooperativas de crédito rural. Pinheiro (2005) define as cooperativas de crédito como instituições financeiras que têm por objeto a prestação de serviços financeiros aos associados, como concessão de crédito, captação de depósitos à vista e a prazo, cheques, prestação de serviços de cobrança, de custódia, de recebimentos e pagamentos por conta de terceiros sob convênio com instituições financeiras públicas e privadas e de correspondente no País, além de outras operações específicas e atribuições estabelecidas na legislação em vigor.
Conforme Silva Filho (2002), as cooperativas de crédito têm objetivos antagônicos às demais corporações financeiras, tendo em vista que são sociedades de pessoas e que não visam essencialmente ao lucro, mas visam ao equilíbrio entre social e economia.

Segundo Gonçalves (2005, p. 1), “as cooperativas de crédito têm também como função promover a desintermediação financeira." Dentre os diferenciais das cooperativas de crédito, o motivo de a entidade ter taxas de captação de recursos diferenciados, mais baixos que os bancos privados, tendem a atender seus princípios e doutrinas o que vai além de atender a maximização dos lucros.

Búrigo (2006b) defende ainda que, as cooperativas não se preocupam em apenas obter benefícios para o seu quadro social, mas sim, estende as suas ações ao máximo de pessoas que integrem o seu segmento.

Ainda Gonçalves (2005, p. 2):

As cooperativas de crédito também diferenciam-se das demais instituições financeiras pelos seus objetivos e pelo público que pretendem atingir, em relação aos serviços financeiros (conta corrente, depósitos de longo prazo, seguros, créditos), buscando garantir maior cidadania.

Atuando com o objetivo de garantir cidadania à população de determinadas regiões de atuação das cooperativas, tem-se que estas passam a ser importantes fontes de desenvolvimento econômico do país.

Pinheiro (2005, p. 7) destaca que "as cooperativas de crédito são um importante instrumento de desenvolvimento em muitos países." Complementa, ainda, que demonstram o potencial de crescimento do cooperativismo de crédito no Brasil.

Pinheiro (2005, p. 8) complementa, ainda, que:

Apesar do potencial de crescimento do segmento no Brasil e da importância que vem adquirindo, é grande o desconhecimento sobre cooperativismo de crédito em nosso país, tanto por parte do público em geral, quanto mesmo por parte de conceituados autores.

No ano de 2009, publicou-se a Lei complementar n. 130 (BRASIL, 2009) a qual dentre outras define que, por meio do art. $2^{\circ}$, as cooperativas de crédito destinam-se, precipuamente, a prover, por meio da mutualidade, a prestação de serviços financeiros a seus 
associados, sendo-lhes assegurado o acesso aos instrumentos do mercado financeiro.

Em 2010, a Resolução 3.859 do Banco Central do Brasil (BRASIL, 2010) altera e consolida as normas relativas à constituição e funcionamento das cooperativas de crédito, prevendo dentre as demais normativas, comprovação das possibilidades de reunião, controle, realização de operações e prestação de serviços na área de atuação pretendida. Acrescenta ainda a apresentação de estudo de viabilidade econômico-financeira abrangendo um horizonte de, no mínimo, três anos de funcionamento.

\subsection{Eficiência e risco de crédito}

As cooperativas têm por finalidade atender aos interesses dos associados, para isso necessita de administração eficiente.

Segundo Silva (2005, p. 28):

As cooperativas atuam em um ambiente de negócios, competindo com outras organizações cooperativas e não cooperativas. Neste ambiente, estas organizações devem apresentar, para conseguir atingir seus objetivos econômicos e sociais, eficiência e eficácia nas suas administrações e assim continuarem atuando no mercado, tendo a cautela de procurar a adequação econômica aos princípios cooperativos.

Para Búrigo (2006a) a eficiência de crédito e viabilidade financeira das cooperativas de crédito deve estar ligada a três situações, sendo elas a origem dos recursos, a relação entre as receitas e despesas e o controle da inadimplência.

A fim de garantir a eficiência de crédito e garantir o capital investido nas cooperativas, é necessário algo mais do que estratégias de captação de recursos e relação de receitas e despesas, é importante também a preocupação com o risco de crédito.

O risco de crédito é algo que está ligado diretamente às empresas que trabalham com ofertas de crédito a consumidores, sejam elas entidades de comércio ou entidades financeiras.

Segundo Brito e Assaf Neto (2008, p. 19):

O processo de avaliação e gerenciamento de risco de crédito em instituições financeiras vem passando por um movimento de revisão ao longo dos últimos anos. Os métodos tradicionais de decisão baseados exclusivamente em critérios julgamentais têm perdido espaço nas atividades de crédito dos Bancos, que buscam instrumentos mais eficazes para mensurar o risco dos tomadores e das carteiras de crédito.

Bancos e demais entidades financeiras tentam cada dia mais buscar instrumentos de análise e gerenciamento de risco de crédito pela importância que o risco tem com o resultado para com as entidades financeiras. Santos, Carmona e Leismann (2010, p. 85):

O risco está presente em boa parte das atividades financeiras e é responsável, pelo menos em parte, por levar a empresa ao sucesso ou fracasso; a concessão de crédito, como atividade financeira, não foge a esta regra: apresenta riscos que devem ser gerenciados tendo em vista a manutenção das atividades empresariais.

Ainda Brito e Assaf Neto (2008, p. 19), “Observa-se uma maior ênfase das instituições na utilização de modelos quantitativos como suporte às decisões de concessão de crédito e à gestão das carteiras".

Segundo Gonçalves (2005, p. 12)

Esses riscos podem ser divididos em risco sistemático e não-sistemático. O sistemático é resultado do sistema político, econômico e social e das características de mercado, como taxas de juros e câmbio, que circundam todas as instituições e impactam-nas de forma indiscriminada. Já o não-sistemático, também denominado risco próprio, é consequência direta dos aspectos financeiros, da administração e do setor de atuação, sendo específico á posição de cada instituição financeira no mercado. Assim, pode-se denominar a análise de risco como o estudo dos eventos que têm efeitos sobre a empresa.

Cabe às entidades de atividade financeira buscar a melhor forma de analisar os riscos que podem vir tanto de aspectos diretos, como a administração e a liberação de crédito como de efeitos sociais e do mercado.

Ainda Gonçalves (2005, p. 18) destaca que "Como os riscos não podem ser totalmente eliminados, a questão é como identificá-los e administrá-los. Para as instituições conseguirem reduzi-lo, elas precisam despender tempo e dinheiro."

Com a identificação e administração dos riscos de crédito, as cooperativas de 
crédito podem amenizar perdas futuras e consequentemente gerar maior riqueza pela redução de perdas.

\section{Metodologia}

A pesquisa se caracteriza como descritiva, documental e quantitativa. Para Vergara (1998, p. 45), a pesquisa descritiva "expõe características de determinada população ou de determinado fenômeno. Pode também estabelecer correlações entre variáveis e definir sua natureza. Não tem compromisso de explicar os fenômenos que descreve, embora sirva de base para tal explicação".

Conforme Martins e Theóphilo (2007), a pesquisa quantitativa consiste em organizar, sumarizar, caracterizar e interpretar dados numéricos coletados, por meio da aplicação de métodos e técnicas estatísticas.

Para Raupp e Beuren (2004), pesquisas quantitativas consistem na aplicação de instrumentos estatísticos, tanto na coleta, quanto em análise dos dados, na qual o procedimento não se aprofunda em saber a realidade dos fatos, mas sim o comportamento geral dos acontecimentos.

Conforme Gil (1996), a pesquisa documental busca retratar as características de um determinado universo. Em relação ao caráter documental, Silva (2010, p. 55) comenta que esta utiliza material que ainda não recebeu tratamento analítico ou que pode ser reelaborado, suas fontes são diversificadas e dispersas.

Em relação à pesquisa documental, foi considerado o ranking das 50 maiores cooperativas de crédito, conforme dados do
BACEN publicados no ano de 2010, no sítio cooperativismo de crédito, que lista as cooperativas ranqueadas pelas variáveis ativos totais, carteira de crédito, depósitos totais, patrimônio líquido e o número de associados. Esses dados foram considerados como fonte primária, uma vez que foram coletados pelos valores absolutos, sem quaisquer tratamentos quantitativos.

Com base nos dados coletados no sítio <www.cooperativismodecredito.com.br>, ano base de 2011, que lista as 50 maiores cooperativas de crédito do Brasil, com auxílio do software estatístico SPSS $® 20$, foi empregada a análise hierárquica dos processos (AHP). A AHP foi exposta por Saaty (1991), conforme destacou Shimizu (2001). A AHP contribui sobremaneira para a pesquisa por considerar sua vasta utilização em situações de escolha entre alternativas com múltiplos critérios, ou ainda, alternativas. Essa técnica alinha com as necessidades da pesquisa, na abordagem quantitativa, uma vez que "caracteriza-se pelo emprego de instrumentos estatísticos, tanto na coleta quanto no tratamento dos dados." (RAUPP; BEUREN, 2004, p. 92).

Quanto à abordagem do problema, nesta pesquisa predominou a análise qualitativa. A abordagem quantitativa destacou o ranking das 50 maiores cooperativas de crédito do Brasil, pela classificação do BACEN, como a manutenção da mesma classificação com a utilização da AHP com base nas mesmas variáveis ou se altera a classificação destacando um novo ranking.

A tabela 1 destaca o ranking das 50 maiores cooperativas de crédito do Brasil, conforme dados do BACEN (dez. 2011). 
Tabela 1 - Ranking das 50 maiores cooperativas de crédito do Brasil

\begin{tabular}{|c|c|c|c|c|c|c|c|}
\hline & Nome da Cooperativa & UF & $\begin{array}{l}\text { Ativos } \\
\text { Totais } \\
\end{array}$ & $\begin{array}{c}\text { Carteira de } \\
\text { Crédito }\end{array}$ & $\begin{array}{l}\text { Total de } \\
\text { Depósitos }\end{array}$ & $\begin{array}{c}\text { Patrimônio } \\
\text { Líquido }\end{array}$ & Associados \\
\hline 1 & SICOOB CREDICITRUS & $\mathrm{SP}$ & 21,73 & 21,10 & 20,80 & 20,33 & 10,83 \\
\hline 2 & SICOOB COCRED & SP & 21,07 & 20,45 & 20,17 & 19,28 & 9,84 \\
\hline 3 & VIACREDI & $\mathrm{SC}$ & 20,84 & 20,35 & 20,40 & 19,34 & 12,14 \\
\hline 4 & SICOOB COOPERFORTE & DF & 20,79 & 20,50 & 20,46 & 19,38 & 11,63 \\
\hline 5 & SICREDI PIONEIRA RS & RS & 20,53 & 19,96 & 20,25 & 18,76 & 11,19 \\
\hline 6 & CREDICOAMO & PR & 20,50 & 19,90 & 19,13 & 19,06 & 9,11 \\
\hline 7 & SICOOB COOPECREDI & $\mathrm{SP}$ & 20,43 & 19,45 & 19,69 & 18,39 & 7,60 \\
\hline 8 & SICREDI UNIÃO PR & $\mathrm{PR}$ & 20,42 & 20,13 & 19,81 & 18,28 & 10,94 \\
\hline 9 & SICREDI UNIÃO RS & RS & 20,42 & 20,17 & 19,88 & 18,60 & 11,60 \\
\hline 10 & SICOOB CREDICOM & MG & 20,39 & 19,16 & 20,25 & 18,08 & 10,51 \\
\hline 11 & SICREDI CATARATAS DO IGUAÇU PR & $\mathrm{PR}$ & 20,37 & 20,11 & 19,94 & 18,22 & 11,00 \\
\hline 12 & SICREDI REGIÃO DOS VALES RS & RS & 20,27 & 19,70 & 19,93 & 18,47 & 10,48 \\
\hline 13 & UNIPRIME PR & $\mathrm{PR}$ & 20,16 & 19,40 & 19,57 & 19,12 & 9,28 \\
\hline 14 & SICREDI CELEIRO DO MT & MT & 20,14 & 19,85 & 19,00 & 18,13 & 9,97 \\
\hline 15 & SICREDI SERRANA RS & RS & 20,09 & 19,56 & 19,83 & 17,90 & 10,95 \\
\hline 16 & SICREDI PLANALTO GAÚCHO RS & RS & 20,02 & 19,72 & 19,01 & 18,26 & 10,33 \\
\hline 17 & UNICRED CENTRO BRASILEIRA GO & GO & 19,97 & 19,55 & 19,68 & 18,26 & 9,04 \\
\hline 18 & SICREDI NORTE RS/SC & RS & 19,96 & 19,60 & 19,55 & 17,86 & 10,90 \\
\hline 19 & SICOOB CREDICOONAI & SP & 19,93 & 19,58 & 18,90 & 18,10 & 9,87 \\
\hline 20 & SICREDI OURO VERDE MT & MT & 19,92 & 19,89 & 18,42 & 18,39 & 9,81 \\
\hline 21 & UNICRED FLORIANÓPOLIS & SC & 19,82 & 19,30 & 19,54 & 18,23 & 9,13 \\
\hline 22 & SICREDI CENTRO-NORTE MT & MT & 19,80 & 19,76 & 18,52 & 17,88 & 9,77 \\
\hline 23 & SICREDI ALTO URUGUAI RS/SC & RS & 19,73 & 19,59 & 19,28 & 17,87 & 10,74 \\
\hline 24 & SICREDI NOROESTE RS & RS & 19,72 & 19,46 & 19,01 & 18,20 & 10,79 \\
\hline 25 & SICREDI ALTOS DA SERRA RS & RS & 19,72 & 19,42 & 19,02 & 18,05 & 10,63 \\
\hline 26 & SICREDI OURO BRANCO RS & RS & 19,71 & 19,40 & 19,31 & 17,68 & 10,60 \\
\hline 27 & SICOOB LESTE CAPIXABA & ES & 19,71 & 19,45 & 19,06 & 18,16 & 10,17 \\
\hline 28 & UNICRED POA & RS & 19,70 & 19,03 & 19,51 & 17,74 & 9,09 \\
\hline 29 & SICREDI REGIÃO CENTRO RS & RS & 19,69 & 19,40 & 19,13 & 17,83 & 10,51 \\
\hline 30 & SICREDI CENTRO-SUL MS & MS & 19,68 & 19,18 & 19,03 & 17,97 & 10,47 \\
\hline 31 & UNICRED JP & $\mathrm{PB}$ & 19,68 & 19,48 & 19,05 & 18,71 & 9,01 \\
\hline 32 & SICOOB MAXICRÉDITO & SC & 19,62 & 19,28 & 19,15 & 17,90 & 10,36 \\
\hline 33 & SICOOB SÃO MIGUEL & SC & 19,61 & 19,09 & 19,04 & 17,73 & 10,34 \\
\hline 34 & UNICRED MT & MT & 19,61 & 19,23 & 19,14 & 18,41 & 8,88 \\
\hline 35 & SICREDI IBIRAIARAS RS & RS & 19,60 & 19,15 & 19,17 & 17,52 & 10,32 \\
\hline 36 & SICREDI VALE DO TAQUARI RS & RS & 19,60 & 19,05 & 19,30 & 17,73 & 10,57 \\
\hline 37 & SICREDI CENTRO SERRA RS & RS & 19,55 & 19,16 & 18,98 & 17,96 & 10,65 \\
\hline 38 & SICOOB SUL-SERRANO & ES & 19,55 & 19,09 & 19,02 & 18,08 & 10,11 \\
\hline 39 & SICOOB AGROCREDI & MG & 19,51 & 19,30 & 18,50 & 17,86 & 9,74 \\
\hline 40 & SICOOB NORTE & ES & 19,50 & 19,23 & 18,69 & 18,08 & 10,15 \\
\hline 41 & SICREDI BOTUCARAI RS & RS & 19,47 & 19,19 & 18,89 & 17,73 & 10,52 \\
\hline 42 & SICREDI ZONA SUL RS & RS & 19,45 & 19,11 & 18,96 & 17,58 & 10,59 \\
\hline 43 & SICOOB SUL & ES & 19,72 & 19,42 & 19,20 & 18,08 & 9,99 \\
\hline 44 & SICOOB COOPMIL & SP & 19,44 & 19,24 & 18,51 & 18,85 & 11,11 \\
\hline 45 & SICREDI UNIÃO METROPOLITANA RS & RS & 19,43 & 18,66 & 19,25 & 17,18 & 10,25 \\
\hline 46 & SICREDI VALE DO RIO PARDO & RS & 19,43 & 19,07 & 18,97 & 17,52 & 10,66 \\
\hline 47 & SICOOB METROPOLITANO & PR & 19,42 & 19,20 & 19,09 & 17,61 & 10,20 \\
\hline 48 & SICREDI SUDOESTE MT & MT & 19,42 & 19,33 & 18,49 & 17,46 & 10,28 \\
\hline 49 & SICOOB CREDI-RURAL & $\mathrm{GO}$ & 19,42 & 19,06 & 18,28 & 18,54 & 8,31 \\
\hline & SICREDI FRONTEIRA PR/SC & PR & 19,41 & 19,35 & 18,87 & 17,53 & 10,25 \\
\hline
\end{tabular}

Fonte: Disponível em: <www.cooperativismodecredito.com.br>. Acesso em: 10 nov. 2012.

Os dados foram normalizados pelo logaritmo Neperiano, de forma que os valores fossem aproximados sem perder a capacidade de tratamento estatístico aplicado, conforme destacado por Carleton (1970), Grace, Harrington e Klein (1998), Fried, Lovell e Yaisawarng (1999). 
Tabela 2 - Matriz de preferências

\begin{tabular}{l|c}
\hline Dados do Ranking BACEN & Ordem de preferências \\
\hline Ativo Total & 0,4 \\
\hline Carteira de Crédito & 0,25 \\
\hline Depósitos Totais & 0,2 \\
\hline Patrimônio Líquido & 0,05 \\
\hline Número de Associados & 0,1
\end{tabular}

Na elaboração da matriz de preferência, foram considerados pesos que representassem um grau de importância entre as variáveis de análise, de forma que sua relação configure na comparação entre as variáveis uma relação de importância. Dessa forma, em relação à soma dos pesos das variáveis, o ativo total figura com $40 \%$ do peso, que o coloca como primeiro no grau de importância. Para as demais variáveis, foi estabelecido procedimento da mesma forma e relação.

Nesta pesquisa, foi apresentada uma matriz de preferência diferente da apresentada por Silva, Fiorentin e Toledo Filho (2011) uma vez que na época o resultado das cooperativas estava influenciado pela relação em que o cooperado (cliente) estava condicionado à situação de sócio da coope- rativa, fato que neste momento, em função da alteração pela resolução n. 3.859/2010, possibilita às cooperativas de crédito aceitar correntistas sem obrigatoriedade de sociedade, de forma que pode ampliar o mix de clientes correntistas.

\section{Análise dos dados}

Com base nos dados apresentados na tabela 1, do ranking das 50 maiores cooperativas de crédito do Brasil, foi considerada matriz de preferência, considerado o disposto apresentado na parte final da metodologia, da tabela 2, foram multiplicados os dados do ranking pela matriz de preferência para a formação do ranking pela AHP, conforme resultado apresentado na tabela 3 .

Tabela 3 - Ranking do BACEN Versus Ranking AHP

\begin{tabular}{l|c|c}
\hline Cooperativa & BACEN & AHP \\
\hline SICOOB CREDICITRUS & 1 & 50 \\
\hline SICOOB COCRED & 2 & 47 \\
\hline VIACREDI & 3 & 49 \\
\hline SICOOB COOPERFORTE & 4 & 48 \\
\hline SICREDI PIONEIRA RS & 5 & 38 \\
\hline CREDICOAMO & 6 & 7 \\
\hline SICOOB COOPECREDI & 7 & 43 \\
\hline SICREDI UNIAO PR & 8 & 45 \\
\hline SICREDI UNIAO RS & 9 & 41 \\
\hline SICOOB CREDICOM & 10 & 44 \\
\hline SICREDI CATARATAS DO IGUAÇU PR & 11 & 32 \\
\hline SICREDI REGIAO DOS VALES RS & 12 & 36 \\
\hline UNIPRIME PR & 13 & 40 \\
\hline SICREDI CELEIRO DO MT & 14 & 37 \\
\hline SICREDI SERRANA RS & 15 & 28 \\
\hline SICREDI PLANALTO GAUCHO RS & 16 & 39 \\
\hline UNICRED CENTRO BRASILEIRA GO & 17 & 18 \\
\hline SICREDI NORTE RS/SC & 18 & \\
\hline
\end{tabular}




\begin{tabular}{|c|c|c|}
\hline Cooperativa & BACEN & AHP \\
\hline SICOOB CREDICOONAI & 19 & 29 \\
\hline SICREDI OURO VERDE MT & 20 & 26 \\
\hline UNICRED FLORIANÓPOLIS & 21 & 17 \\
\hline SICREDI CENTRO NORTE MT & 22 & 19 \\
\hline SICREDI ALTO URUGUAI RS/SC & 23 & 35 \\
\hline SICREDI NOROESTE RS & 24 & 33 \\
\hline SICREDI ALTOS DA SERRA RS & 25 & 31 \\
\hline SICREDI OURO BRANCO RS & 26 & 32 \\
\hline SICOOB LESTE CAPIXABA & 27 & 27 \\
\hline UNICRED POA & 28 & 6 \\
\hline SICREDI REGIÃO CENTRO RS & 29 & 30 \\
\hline SICREDI CENTRO-SUL MS & 30 & 23 \\
\hline UNICRED JP & 31 & 8 \\
\hline SICOOB MAXICRÉDITO & 32 & 24 \\
\hline SICOOB SÃO MIGUEL & 33 & 16 \\
\hline UNICRED MT & 34 & 2 \\
\hline SICREDI IBIRAIARAS RS & 35 & 18 \\
\hline SICREDI VALE DO TAQUARI RS & 36 & 22 \\
\hline SICREDI CENTRO SERRA RS & 37 & 21 \\
\hline SICOOB SUL-SERRANO & 38 & 12 \\
\hline SICOOB AGROCREDI & 39 & 3 \\
\hline SICOOB NORTE & 40 & 9 \\
\hline SICREDI BOTUCARAI RS & 41 & 15 \\
\hline SICREDI ZONA SUL RS & 42 & 14 \\
\hline SICOOB SUL & 43 & 25 \\
\hline SICOOB COOPMIL & 44 & 20 \\
\hline SICREDI UNIAO METROPOLITANA RS & 45 & 4 \\
\hline SICREDI VALE DO RIO PARDO & 46 & 13 \\
\hline SICOOB METROPOLITANO & 47 & 11 \\
\hline SICREDI SUDOESTE MT & 48 & 5 \\
\hline SICOOB CREDI-RURAL & 49 & 1 \\
\hline SICREDI FRONTEIRA PR/SC & 50 & 10 \\
\hline
\end{tabular}

Com base nos dados da tabela 3, ficaram ressaltadas as alterações na classificação das cooperativas, pela metodologia do BACEN, considerada a tabela 2 , da matriz de preferências, que alterou significativamente a posição de classificação no ranking apresentado pela AHP. Apenas as cooperativas classificadas na sétima e na vigésima sétima posições não sofreram alterações.

Das dez primeiras classificadas pelos critérios (graus de preferência) do BACEN, quando consideradas no ranking apresentado pela $\mathrm{AHP}$, oito cooperativas passaram para depois da quadragésima primeira posição. Já, das dez últimas classificadas pelo BACEN, o ranking apresentado pela AHP reposicionou quatro delas entre as dez melhores cooperativas de crédito, com base na matriz de preferência presentemente utilizada.

A pesquisa de Silva, Fiorentin e Toledo Filho (2011) destacou alterações de menor grau, com apenas duas posições sendo divergentes do ranking estabelecido pelo BACEN. fato que não aconteceu nesta pesquisa.

Concernente aos resultados apresentados, o objeto do estudo foi de análise do 
ranking anteriormente utilizado, porém, tampouco foi foco de negação a classificação anterior, mas que, por se tratar de estudo que envolve o estabelecimento por parte do pesquisador na preferência no peso das variáveis, as alterações podem acontecer em maior ou menor grau. Nesta pesquisa, ficou evidente a troca de posição pelas diferenças estabelecidas para o tratamento dos dados, como de maior grau de alterações de posição.

Posteriormente, como ponto de confirmação aos resultados apresentados, foi aplicado o teste do coeficiente de correlação de Kendall, com utilização do software estatístico, da forma apresentada no tópico seguinte.

\section{Coeficiente de correlação de Kendall}

A aplicação do teste de correlação de Kendall foi utilizada com predisposição afirmativa aos resultados conseguidos, com base na classificação do BACEN e do ranking no uso da AHP, tendo como objeto o exame dos resultados apresentados. O teste de coeficiente de Kendall já foi utilizado em vasta gama de pesquisa, dentre elas, os estudos na contabilidade apresentados por Cunha e Beuren (2006, p. 82) utilizaram em testes de auditoria, bem como o de Silva, Fiorentin e Toledo Filho (2011) para o ranking das cooperativas.

Destacamos ainda, que a pesquisa de Fonseca, Martins e Toledo (1995, p. 48) esclarece, também, "o coeficiente de Kendall, simbolizado pela letra grega $\tau$ (tau), fornece uma medida mais satisfatória de associação entre classificações, principalmente quando o número de relações é muito grande". O coeficiente foi representado como:

$$
\tau=\frac{s}{\frac{n(n-1)}{2}}
$$

Onde: $\tau=$ Associação das Classificações Requeridas;

$S=$ resultado da relação das ordens encontradas nos possíveis pares de ordenação;

$\mathrm{N}$ = número de etapas.

$\mathrm{O}$ " $\mathrm{S}$ " é calculado para destacar a relação dos pares possíveis, que representa o teste da classificação apresentada pelo BACEN e o ranking apresentado pela AHP.

Tabela 4 - Correlação de Kendall

\begin{tabular}{l|l|c|c}
\hline \multicolumn{2}{c|}{} & BACEN & AHP \\
\hline \multirow{3}{*}{ BACEN } & Coeficiente de correlação & 1,000 & $-0,678^{* *}$ \\
\cline { 2 - 4 } & Sig. (2-tailed) &. & 0,000 \\
\cline { 2 - 4 } & $\mathrm{N}$ & 50 & 50 \\
\hline \multirow{3}{*}{ AHP } & Coeficiente de correlação & $-0,678^{* *}$ & 1,000 \\
\cline { 2 - 4 } & Sig. (2-tailed) & 0,000 & 50 \\
\cline { 2 - 4 } & $\mathrm{N}$ & 50 & 5 \\
\hline \multirow{2}{*}{$* *$ Nível de significância da Correlação 0,01\%. }
\end{tabular}

A correlação de Kendall apresentou divergência entre os rankings do BACEN e o resultado da classificação pelo método AHP. $\mathrm{O}$ ranking apresentado pela AHP diverge a tal ponto que tendem a inverter as posições em relação ao apresentado pelo BACEN, com $-0,678$.

Os resultados são divergentes a tal ponto que, conforme apresentado na tabela 3 , destaca que, das 50 cooperativas de crédito, apenas duas cooperativas, a sétima e a vigésima sétima se mantiveram na mesma posição. As demais cooperativas alteraram suas posições quando utilizada a AHP.

\section{Considerações finais}

O presente estudo realizou-se com a premissa de que as cooperativas de crédito com maior ativo devem estar classificada como primeira colocada em depósitos totais, patrimônio líquido e operações de crédito.

Com o objetivo de analisar a eficiência e o posicionamento do ranking das cooperativas de crédito no Brasil, esta pesquisa utilizou a classificação feita pelo BACEN, com ano base de 2010, com as variáveis ativos totais, créditos totais, depósitos totais, patrimônio líquido e o volume de cooperados, que nesse 
conjunto classificou as 50 maiores cooperativas do Brasil.

Esses dados foram base para este estudo, em que se utilizou da técnica multivariada de dados, Análise Hierárquica de Dados (AHP), em que foram classificadas em função da matriz de preferência. Com isso foi listado o ranking original das 50 maiores cooperativas de crédito brasileiras segundo o Banco Central do Brasil (BACEN) e apurado o ranking com base na análise hierárquica de processos.

Esse ranking da AHP em relação ao original apresentado pelo BACEN destaca uma divergência na classificação final, pois as alterações que validavam o ranking foram altíssimas, com permanência de, apenas, duas cooperativas de crédito na posição original e no ranking da AHP. Esse fato destaca a disparidade entre as classificações, em que do ranking original foram utilizadas as variáveis puras e, no ranking da AHP, com base na matriz de preferência, as posições se alteraram de forma demasiada. Ainda, a matriz de preferência foi estabelecida com característica principal de prioridade na ordem em que as variáveis listadas.

Contudo o número de cooperados foi classificado com grau de preferência menor pois o associado está desvinculado de contribuição na forma de cooperado, pela alteração na legislação, posicionamento diferente do apresentado na pesquisa de Silva, Fiorentin e Toledo Filho (2011) em que as alterações na posição de classificação, também com uso da AHP, foram de apenas duas cooperativas de crédito.

Como sugestão de pesquisas propõe-se ampliar mais o número de cooperativas de crédito, além da possibilidade de estudo com as cooperativas de microcrédito, ainda com pouca exploração no tema.

\section{Referências}

ARCHER, S. H. The structure of management decision theory. The Academy of Management Journal, New York, USA, v. 7, n. 4, p. 269-287, Dec. 1964.

BACCARINI, D.; ARCHER, R. The risk ranking of projects: a methodology. International Journal of Project Management, Philadelphia, USA, v. 19, n. 1, p. 139-145, 2001.

BRASIL. Lei n. 4.595 de 31 de dezembro de 1964. Dispõe sobre a Política e as Instituições Monetárias, Bancárias e Creditícias, Cria o Conselho Monetário Nacional e dá outras providências. Disponível em: <http:/ / www.
planalto.gov.br/ccivil_03/leis/L4595.htm>. Acesso em: 20 nov. 2011.

. Lei Complementar n. 130, de 17 de abril de 2009. Dispõe sobre o Sistema Nacional de Crédito Cooperativo e revoga dispositivos das Leis nos 4.595, de 31 de dezembro de 1964, e 5.764, de 16 de dezembro de 1971. Disponível em: <http:/ / www.planalto.gov.br>. Acesso em: 25 maio 2012.

. Resolução n. 3.859 de 27 de maio de 2010. Altera e consolida as normas relativas à constituição e ao funcionamento de cooperativas de crédito. Disponível em: <www.cooperativismodecrédito.com.br>. Acesso em: 25 maio 2012.

BRITO, G. A. S.; ASSAF NETO, A. Modelo de classificação de risco de crédito de empresas. Revista Contabilidade E Finanças, São Paulo, v. 19, n. 46, p. 18-29, jan./ abr. 2008.

BÚRIGO, F. L. Finanças e solidariedade: o cooperativismo de crédito rural solidário no Brasil. Estudos Sociedade e Agricultura (UFRJ), Rio de Janeiro, v. 14, n. 2, p. 312-349, dez. 2006a.

Finanças e solidariedade: uma análise do cooperativismo de crédito rural solidário no Brasil. 2006. 374f. Tese (Doutorado em Sociologia Política) - Universidade Federal de Santa Catarina, Florianópolis, 2006b.

CARLETON, W. T. An Analytical Model for LongRange Financial Planning. The Journal of Finance, Malden, USA, v. 25, n. 2, p. 291-315, 1970.

COOPERATIVISMO de Crédito. Disponível em: <www. cooperativismodecredito.com.br>. Acesso em: 20 nov. 2011.

CUNHA, P. R.; BEUREN, I. M. Técnicas de amostragem utilizadas nas empresas de auditoria independente estabelecidas em Santa Catarina. Revista Contabilidade $\mathcal{E}$ Finanças, São Paulo, v. 17, n. 40, p. 67-86, jan./abr. 2006.

DAUTEN, C. A. A fresh approach to the place of consumer credit in economic and financial thinking. The Journal of Finance, Malden, USA, v. 9, n. 2, p. 111-123, May 1954.

DAYSON, K.; CARBONI, B. J.; KICKUL, J.; CALDERON, M.; GARRIDO, S. R. Introduction. In. CARBONI, B. J. (Org.). Handbook of microcredit in Europe: social inclusion through microenterprise development. Cheltenham, UK: MPG Books Groupl, 2010.

FAMA, E. F. Components of Investment Performance. The Journal of Finance, Malden, USA, n. 27, v. 3, p. 551567, 1972.

FONSECA, J. S.; MARTINS, G. A.; TOLEDO, G. L. Estatística aplicada. 2. ed. São Paulo: Atlas, 1995.

FRIED, H. O.; LOVELL, C. A. K.; YAISAWARNG, S. The impact of mergers on credit union service provision. Journal of Banking \& Finance, Santa Clara, USA, v. 23, p. 367-386, 1999.

GIL, A. C. Métodos e técnicas de pesquisa social. 5. ed. São Paulo: Atlas, 1996.

GONÇALVES, R. M. L. Condicionantes do risco de liquidez em cooperativas de economia e crédito mútuo do estado de Minas Gerais. 2005. 118f. Dissertação (Mestrado em Economia Aplicada) - Programa de Pós-graduação em 
Economia Aplicada, Universidade Federal de Viçosa, Viçosa, MG, 2005.

GRACE, M. F.; HARRINGTON, S. E.; KLEIN, R. W. Risk-based capital and solvency screening in propertyliability insurance: hypotheses and empirical tests. The Journal of Risk and Insurance, Pennsylvania, USA, n. 65, v. 2, p. 213-243, 1998.

HORRIGAN, J. O. A short history of financial ratio analysis. The Accounting Review, Pittsburgh, USA, n. 43, v. 2, p. 284-294, 1968.

LAZZARINI, S. G.; BIALOSKORSKI NETO, S.; CHADDAD, F. R. Decisões financeiras em cooperativas: fontes de ineficiência e possíveis soluções. Gestão $e$ Produção (UFSCAR), São Carlos, SP, v. 6, n. 3, p. 257268, dez. 1999.

MARTINS, G. A.; THEÓPHILO, C. R. Metodologia da investigação científica para ciências sociais aplicadas. São Paulo: Atlas, 2007.

PINHEIRO, M. A. H. Cooperativas de crédito: história da evolução normativa no Brasil. Brasília: BCB, 2005. 6. ed. Brasília: BCB, 2008.

RAUPP, F. M.; BEUREN, I. M. Caracterização da pesquisa em Contabilidade. In. BEUREN, I. M. (Org.). Como elaborar trabalhos monográficos em contabilidade: teoria e prática. 2 ed. São Paulo: Atlas, 2004.

ROBBINS, S.; FOSTER JR, E. Profit-planning and the finance function. The Journal of Finance, Malden, USA, v. 12, n. 4, p. 451-467, Dec., 1957.

SAATY, T. L. Método de análise hierárquica. São Paulo: Makron Books, 1991.

SANTOS, K. F.; CARMONA, C. U. M.; LEISMANN, E. L. Gerenciamento do risco de crédito em micro e pequenas empresas: recortes teórico-empíricos. Revista da Micro e Pequena Empresa, Campo Limpo Paulista, SP, v. 3, n. 3, p. 83-95, 2010.
SHIMIZU, T. Decisão nas organizações. São Paulo: Atlas, 2001.

SILVA, E. S. A eficiência econômica e social em cooperativas do setor pecuário em Pernambuco. Custos e @ gronegócio on line, Recife, PE, v. 1, n. 2, p. 25-44, 2005.

SILVA, A. C. R. Metodologia da pesquisa aplicada à contabilidade: orientações de estudos, projetos, artigos, relatórios, monografias, dissertações, teses. 3. ed. São Paulo: Atlas, 2010.

SILVA, T. P.; FIORENTIN, M.; TOLEDO FILHO, J. R. Análise da eficiência e do ranking das dez maiores cooperativas de crédito do Brasil. Espacios, Caracas, v. 2, n. 32, p. 29-46, 2011.

SILVA FILHO, G. T. Avaliação de desempenho em cooperativas de crédito: uma aplicação do modelo de gestão econômica - GECON. Organizações Rurais $\mathcal{E}$ Agroindustriais, Lavras, MG, v. 1, n. 4, p. 266-282, 2002.

SILVA, T. P. Risco positivo na atividade de crédito que otimiza o desempenho econômico-financeiro de cooperativas de crédito. 2012. 203p. Tese (Doutorado em Ciências Contábeis e Administração) - Programa de Pós-Graduação em Ciências Contábeis da Universidade Regional de Blumenau, Blumenau, SC, 2012.

SOARES, M. M.; MELO SOBRINHO, A. D. Microfinanças: o papel do Banco Central do Brasil e a importância do cooperativismo de crédito. Brasília: BCB, 2007.

TURNOVSKY, S. J. Financial structure and the theory of production. The Journal of Finance, Malden, USA, v. 25, n. 5, p. 1061-1080, Dec., 1970.

VERGARA, S. C. Projeto e relatórios de pesquisa em administração. São Paulo: Atlas, 1998.

ZHOU, L.; LAI, K. K. Multi-agent ensemble models based on Weighted Least Square SVM for credit risk assessment. Global Congress on Intelligent Systems, Hong Kong, China, 19-21 May 2009. v. 3. 